\title{
An MRI, SPECT and neuropsychological study of a patient presenting with Capgras syndrome
}

\author{
J. Mackie, K.P. Ebmeier and R.E. O'Carroll \\ MRC Brain Metabolism Unit, Royal Edinburgh Hospital, Morningside Park, \\ Edinburgh EH10 5HF, UK
}

Correspondence to: R.E. O'Carroll at above address

\begin{abstract}
A 25 year old male patient presented with the "delusion of doubles" (Capgras syndrome). The patient underwent detailed neuropsychological, single photon emission computed tomography (SPECT) and magnetic resonance imaging (MRI) investigations. The neuropsychological results indicated that he was of average premorbid intelligence, which was consistent with current estimates, and had a degree of everyday memory dysfunction which was below norms derived from healthy controls, but was average relative to schizophrenic norms. He demonstrated average or above average recognition memory for patterns, spaces or words, but exhibited a marked and disproportionate impairment in face recognition, performing at the 5th percentile. In addition he demonstrated markedly impaired performance on various tests of executive function. MRI scanning revealed no focal abnormality; slight ventricular dilatation was noted. SPECT scanning, however, revealed marked right/left asymmetries of tracer uptake, restricted to occipital and calcarine cortices. These asymmetries were well outside those derived from a cohort of healthy controls. These results are discussed in relation to current neuropsychological models of Capgras syndrome.
\end{abstract}

Keywords: Asymmetry - Delusion - Executive - Face recognition - Memory - Neuropsychology - SPECT

\section{INTRODUCTION}

The "delusion of doubles", first reported by Capgras and Reboul-Lachaux in 1923, describes the belief that close relatives have been replaced by identical imposters. The syndrome is of more than phenomenological interest, as sufferers occasionally resort to acts of violence against the perceived imposter (Thompson and Swan, 1993). A fuller understanding of this intriguing condition is required. In the past few years Capgras syndrome has been the subject of intense neuropsychological study. Investigators have consistently reported marked and specific impairments of face recognition, (familiar and unfamiliar), despite average or above average spatial and pattern recognition, word recognition and intelligence (e.g. Wright et al., 1993; Young et al., 1993). Additionally, Ellis et al. (1993) reported that in a tachistoscopic face recognition paradigm, patients exhibited a reversal of the usual left visual field/right hemisphere advantage.

A currently influential neuropsychological model of Capgras syndrome assumes that sufferers have an intact primary ventral route to face recognition (occipital-temporal-orbitofrontal), but that a secondary dorsal route (occipital-parietal-dorsolateral frontal), which gives the face its emotional significance, becomes dysfunctional (Ellis and Young, 1990).
These authors hypothesize that Capgras patients recognize the familiar person, but that this is not associated with the appropriate emotional valence. To explain the observation that Capgras patients also appear to have marked problems with unfamiliar face recognition, Young et al. (1993) proposed that substantive parts of the pathways which imbue visual stimuli with affective significance are close to those involved in visual recognition, so that one would expect few brain lesions to compromise only emotional reactions to visual stimuli without also affecting other visual functions.

Capgras syndrome is commonly associated with other paranoid and persecutory symptoms (Malloy et al., 1992; Silva and Leong, 1992). Patients with persecutory delusions tend to attribute negative events to external rather than internal causes (Kaney and Bentall, 1989). Incorporating these observations, Wright et al. (1993) propose that the persecutory delusions and suspiciousness associated with Capgras syndrome contributes to the patients' mistaking a change in themselves for a change in others ("They are imposters").

The functional neuroanatomy of Capgras syndrome remains unidentified. McCarthy and Warrington (1990) suggest that lesions of right posterior 
cortex are most often associated with face recognition difficulties. Specifically with regard to Capgras syndrome, the neuroimaging evidence is extremely limited; some reports document fronto-temporal abnormalities (Lewis, 1987; Joseph et al. 1990; Durani et al. 1991; Kim, 1991; Malloy et al., 1992; Signer, 1992) whereas Kumar (1987) and Forstl et al. (1991) claimed that marked cerebral lesions and specific neuropsychological deficits are not required for the development of the Capgras syndrome, and that it is often associated with global cerebral atrophy in the elderly. Most of the studies to date have used relatively crude anatomical images derived from computed tomography (CT) scans. Ellis and Young (1990) recently made the plea that: "The next step must surely be to derive routinely, in cases of delusional misidentification syndromes, high-quality brain images that should assist in classification. In time it should be possible to correlate particular syndromes with damage to specific brain areas" (p. 247). Similar recommendations have been made by Bhatia (1990) and Collins et al. (1990).

Since the delusion of doubles is often reversible, this may imply a functional (as opposed to structural) abnormality which normalizes on recovery. This could therefore be identified with functional neuroimaging during the ill state. In this report we document neuropsychological, single photon emission computed tomography (SPECT) and magnetic resonance imaging (MRI) findings in a young man presenting with Capgras syndrome. We predicted specific face recognition deficits associated with hypoperfusion of right posterior cortex.

\section{CASE REPORT}

The patient, a man in his mid-twenties, presented to the emergency psychiatric services, accompanied by his mother and brother. He complained that his flat had been "bugged" by his parents, that he was the target of a conspiracy, and that he was being spied upon and followed. He could hear voices, usually in his flat, talking amongst themselves and commenting on him by repeatedly asking "is that it?" He reported having heard a noise like a "walkie-talkie" coming from his motor bike, which he then dismantled in an attempt to find the source of the noise. On admission he did not accept that he had an illness. He refused all oral medication and was eventually given a depot antipsychotic while on a treatment order. After 18 days, he was considered well enough to be transferred to the open sector ward. He was still suspicious, replying "don't know" to any questions he considered intrusive. $\mathrm{He}$ admitted to having had "bad thoughts" at admission, but stated that he was no longer troubled by them.

Approximately 1 week after transfer to the sector ward, he began to talk more openly about his recent experiences. He stated that over the past year people had "changed", particularly his parents and close relatives. They would often leave a room and an almost identical imposter would return. He could identify imposters, because they were marginally shorter and wider than the real individuals. Such changes would occur repeatedly, often many times within the same day. The process angered him considerably, and he felt it was carried out in an attempt to "get at" him.

He was born with a forceps delivery; there were no problems during pregnancy. He was described by his mother as hyperactive, always having a poor relationship with teachers. However, he was also outgoing and happy, and had many friends. He left school at 16 with 4 "O" levels. He embarked on an electrical engineering course at college, but dropped out at an early stage. At the age of 19 he had a year-long relationship with a girl-friend, however she moved away and the relationship broke down. He attended various courses, e.g. on motor-cycle maintenance. His most recent employment was as a landscape gardener, 18 months prior to admission, but he had been unemployed since then. At the time of admission he was living alone in a flat owned by his parents, supported by social security. He has two older brothers who are both well, and one younger brother who has a diagnosis of schizophrenia, who absconded from a psychiatric hospital and has not been heard of for over a year.

\section{INVESTIGATIONS}

The patient was comprehensively assessed during his admission. At the time of investigation he fulfilled DSM-III-R criteria for schizophrenia (paranoid type). His total score on the Brief Psychiatric Rating Scale was 26 , scoring highest on "unusual thought content" and "blunted affect" (both 5/6). On the Manchester rating scale (0-4; Krawiecka et al., 1977) his scores were Depressed $=0, \quad$ Anxious $=2$, Delusions $=4$, Hallucinations $=0$, Incoherence $=$ 1, Poverty of speech $=3$, Flattened affect $=3$, Incongruous affect $=0$, Psychomotor retardation $=1$. He stated that he was feeling much better and was no longer troubled by the "bad thoughts" that had led to his admission. However, on closer questioning he admitted that the "change-around game" with his family was continuing exactly as before, but that he was trying to ignore it, and he was not letting others 
know that it continued to annoy him. At this time he was being treated with fluphenazine $25 \mathrm{mg}$ fortnightly.

His premorbid intelligence, assessed using the National Adult Reading Test (NART) (Nelson and Willison, 1991), was average at 97, which agreed well with current intelligence measures taken from selected sub-tests from the WAIS-R (Wechsler, 1981; Similarities age scaled score $=9$, Picture Completion $=10$, and Digit Span = 11). Relative to healthy controls his everyday memory functioning was moderately impaired, scoring 16/24 on the Rivermead Behavioural Memory Test (Wilson et al., 1989), though not particularly impaired relative to other schizophrenic patients (McKenna et al. 1990; Duffy and O'Carroll, 1994). He scored 91 on the Stroop Test (Trenerry et al., 1988) which gives a percentile score of 10 and a "probability of brain damage" of 0.70 . FAS verbal fluency was 22 , over 2 standard deviations below the age-controlled mean (Spreen and Strauss, 1991). He was also assessed on a variety of the subtests from the Cambridge Automated Neuropsychological Testing Assessment Battery (CANTAB; Sahakian and Owen, 1992). His pattern recognition score of $19 / 24$ was at the 15 th percentile whereas his spatial recognition was perfect at 20/20 (95th percentile). His recognition memory at various delays was average at 10/10 (simultaneous), 7/10 (0 s), 7/10 (4 s) and 9/10 (12 s). On executive tasks he displayed marked impairments. On the CANTAB Tower of London planning task he took many more moves than necessary for problem solution: mean number of moves above minimum number for the three move task was 0.5 , for the four move task was 2.25 , and for the five move task was 3.75. His set-shifting ability was assessed using the CANTAB intra-dimensional/extra-dimensional shift task, however, the test aborted at stage 6 , indicating that he took over 50 trials without achieving criterion at the first intra-dimensional shift. Finally, his recognition memory for faces and words was assessed using Warrington's Recognition Memory Test (Warrington, 1984) where he scored $47 / 50$ (50th percentile) for words and $37 / 50$ (5th percentile) for faces.

To summarize the neuropsychological findings, he was of average premorbid intelligence which was consistent with current estimates, with a degree of everyday memory functioning commonly observed in schizophrenia, with average or above average recognition memory for patterns, spatial locations and words, but with a disproportionate impairment in face recognition, associated with consistently impaired performance on tests of executive functioning.

\section{NEUROIMAGING}

The MRI scan (1 Tesla) showed mild ventricular dilatation but no focal changes. SPECT was performed using a single slice 12 detector scanner (SME-Neuro 900 version 2.8; Strichman, Medfield, MA) with a resolution of $7.5 \mathrm{~mm}$ (full width, half maximum). The patient underwent cannulation of an arm vein and was allowed to rest for $10 \mathrm{~min}$ before being injected with $500 \mathrm{MBq}$ of ${ }^{99 \mathrm{~m}} \mathrm{Tc}$-Exametazime. During the injection, he was lying comfortably on the imaging table, with eyes covered, ears unplugged and environmental noises kept to a minimum for 5 min after the start of the injection. No obvious lesion was detected on visual inspection of the scan, but there was a slight asymmetry noted in calcarine and occipital cortex, with a reduction of uptake in the right side. Two transverse slices parallel to and 4 and $6 \mathrm{~cm}$ above the orbitomeatal line were selected for quantitative analysis. Further details of the positioning method as well as the analysis of the imaging data have been described previously (Ebmeier et al., 1991). Regional tracer uptake for the patient was normalized by computing regional left-right ratios and whole-slice ratios, compared with a group of 41 healthy volunteers with a mean age of 51 (with standard deviation of 18 years). These subjects were examined with an identical imaging protocol. Using regression with binomials of age, regional tracer uptake was modelled and recalculated for the age of the patient examined.

Left-right ratios of calcarine and occipital regions in both slices were well outside the $95 \%$ confidence limits of the control group data, (i.e. age-adjusted mean \pm 2 S.D., see Table I). Interestingly, this was reflected in increased left occipital and calcarine whole-slice uptake ratios, but not in significant reductions on the right side. A significant increase in tracer uptake was also observed in right putamen.

\section{DISCUSSION}

In this single case study of a young man presenting with Capgras syndrome, we have identified a markedly abnormal neuropsychological profile characterized by a specific impairment of face recognition together with a dysexecutive syndrome. There was no detectable structural lesion identified using high quality MRI. However, functional neuroimaging using SPECT revealed marked abnormalities of posterior brain function. We predicted hypoperfusion of right posterior cortex, and left-right ratio calculations revealed a highly statistically significant abnormality (relative to healthy controls) restricted to posterior 
TABLE I. Left-right (L-R) indices and whole slice (WS) normalized data for posterior regions of interest (if the uptake ratio was correlated with age, agecorrected means and confidence limits are given in bold)

\begin{tabular}{lcccc}
\hline Region & Case & $\begin{array}{c}\text { Control } \\
\text { mean }\end{array}$ & $\begin{array}{c}\text { Lower } \\
\text { confidence } \\
\text { limit }\end{array}$ & $\begin{array}{c}\text { Higher } \\
\text { confidence } \\
\text { limit }\end{array}$ \\
\hline Lower slice & $12.9^{*}$ & -1.9 & -14.7 & 11.0 \\
L-R calcarine & $14.9^{*}$ & $-\mathbf{1 . 5}$ & -10.2 & $\mathbf{7 . 2}$ \\
L-R occipital & $138.0^{*}$ & 118.1 & 102.0 & 134.2 \\
L calcarine (WS) & 121.3 & 120.3 & 103.9 & 136.7 \\
R calcarine (WS) & $125.6^{*}$ & $\mathbf{1 0 3 . 0}$ & $\mathbf{9 0 . 6}$ & $\mathbf{1 1 4 . 8}$ \\
L occipital (WS) & 108.2 & 10.71 & 94.8 & 119.5 \\
R occipital (WS) & & & & \\
& & & & \\
Higher slice & $14.1^{*}$ & 1.5 & -9.2 & 12.1 \\
L-R occipital & 116.2 & 110.2 & 95.9 & 124.5 \\
L occipital (WS) & 100.9 & $\mathbf{1 0 5 . 1}$ & $\mathbf{9 0 . 8}$ & $\mathbf{1 1 9 . 4}$ \\
R occipital (WS) & & &
\end{tabular}

$L-R$ indices were calculated using the formula:

$$
L-R=\frac{L-R}{L+R} \times 200
$$

WS normalized data were calculated using the formula:

$$
\frac{\text { region of interest count density }}{\text { whole slice count density }} \times 100
$$

* Outwith $95 \%$ confidence intervals derived from healthy controls

cortex. Contrary to our prediction however, this was the result of hyperperfusion of left occipital and calcarine cortex. Of course, SPECT data provide relative, not absolute quantification, and the most parsimonious interpretation is to conclude that a marked asymmetry of posterior brain function was observed in the present case. In attempting to integrate the SPECT data with the neuropsychological results, the posterior cortex abnormalities of metabolism can readily be reconciled with the observed impairment in face recognition (McCarthy and Warrington, 1990). The marked deficit on all executive tasks is more difficult to explain. Such tasks are considered to be particularly sensitive to lesions of frontal cortex, however, relative to healthy controls, no anterior abnormalities were detected in the present case, using either MRI or SPECT. This is consistent with our results in medicated schizophrenic patients who did not show any reduction in tracer uptake in lateral prefrontal cortex (Ebmeier et al., 1994). Rather than operating within the constraints of a "focal lesion for a focal cognitive test" it is perhaps more appropriate to consider functional impairments of distributed pathways. Adopting the Ellis and Young (1990) model that Capgras syndrome is associated with a dysfunctional dorsal route to face recognition (occipital-parietal-dorsolateral-frontal), in the present case we have good evidence of abnormalities of occipital brain function, and it is not unreasonable to speculate that the consequent dysfunctional projections to frontal cortex lead to impairment on tests of executive function.

\section{Acknowledgements}

We thank the Wellcome Trust for support, and Norma Brearley for her careful preparation of the manuscript.

\section{REFERENCES}

Bhatia MS (1990) Capgras syndrome in a patient with migraine. British Journal of Psychiatry, 157, 917-918.

Collins MN, Hawthorne ME, Gribbin $\mathrm{N}$ and Jacobson $\mathrm{R}$ (1990) Capgras' syndrome with organic disorders. Postgraduate Medical Journal, 66, 1064-1067.

Duffy L and O'Carroll R (1994) Memory impairment in schizophrenia-a comparison with that observed in the alcoholic Korsakoff syndrome. Psychological Medicine, 24, 155-165.

Durani SK, Ford R and Sajjad SHA (1991) Capgras syndrome associated with a frontal lobe tumour. Irish Journal of Psychological Medicine, 8, 135-136.

Ebmeier KP, Dougall NJ, Austin M-PV, Murray CL, Curran SM, O'Carroll R, Moffoot APR, Hannan J and Goodwin GM (1991) The split-dose technique for the study of psychological and pharmacological activation with the cerebral blood flow marker Exametazime and Single Photon Emission Computed Tomography 
(SPECT): reproducibility and rater reliability. International Journal of Methods in Psychiatric Research, 1, 27-38.

Ebmeier KP, Lawrie SM, Blackwood DHR, Johnstone EC and Goodwin GM (1994). Reduced activity in anterior cingulate in patients with schizophrenia: assessment at rest with high resolution single photon emission tomography. Submitted for publication.

Ellis HD and Young AW (1990) Accounting for delusional misidentifications. British Journal of Psychiatry, 157, 239-248.

Ellis HD, De Pauw KW, Christodoulou GN, Papageorgiou L, Milne AB and Joseph AB (1993) Responses to facial and non-facial stimuli presented tachistoscopically in either or both fields by patients with the Capgras delusion and paranoid schizophrenics. Journal of Neurology, Neurosurgery and Psychiatry, 56, 215-219.

Forstl H, Almeida OP and Iacoponi E (1991) Capgras delusion in the elderly: The evidence for a possible organic origin. International Journal of Geriatric Psychiatry, 6, 845-852.

Joseph AB, O'Leary DH and Wheeler HG (1990) Bilateral atrophy of the frontal and temporal lobes in schizophrenic patients with Capgras syndrome: A case-control study using computed tomography. Journal of Clinical Psychiatry, 51, 322-325.

Kaney S and Bentall RP (1989) Persecutory delusions and attributional style. British Journal of Medical Psychology, 62, 191-198.

Kim E (1991) A post-ictal variant of Capgras' syndrome in a patient with a frontal meningioma. A case report. Psychosomatics, 32, 448-451.

Krawiecka M, Goldberg D and Vaughn M (1977) A standardised psychiatric assessment scale for rating psychotic patients. Acta Psychiatrica Scandinavica, 55, 299-308.

Kumar V (1987) Capgras syndrome in a patient with dementia. British Journal of Psychiatry, 150, 251.

Lewis SW (1987) Brain imaging in a case of Capgras' syndrome. British Journal of Psychiatry, 150, 117-121.

Malloy P, Cimino C and Westlake R (1992) Differential diagnosis of primary and secondary Capgras delusions. Neuropsychiatry, Neuropsychology and Behavioral Neurology, 5, 83-96.
McCarthy RA and Warrington EK (1990) Cognitive Neuropsychology: A Clinical Introduction. Academic Press, London.

McKenna PJ, Tamlyn D, Lund CE, Mortimer AM, Hammond S and Baddeley AD (1990) Amnesic syndrome in schizophrenia. Psychological Medicine, 20, 967-972.

Nelson HE and Willison JR (1991) The Revised National Adult Reading Test-Test Manual. NFER-Nelson.

Sahakian BJ and Owen AM (1992) Computerized assessment in neuropsychiatry using CANTAB: discussion paper. Journal of the Royal Society of Medicine, 85, 399402.

Signer SF (1992) Capgras symptom and delusions of reduplication in neurologic disorders. Neuropsychiatry, Neuropsychology and Behavioral Neurology, 5, 138-143.

Silva JA and Leong GB (1992) The Capgras syndrome in paranoid schizophrenia. Psychopathology, 25, 147-153.

Spreen O and Strauss E (1991) A Compendium of Neuropsychological Tests. Oxford University Press, Oxford.

Thompson AE and Swan M (1993) Capgras' syndrome presenting with violence following heavy drinking. British Journal of Psychiatry, 162, 692-694.

Trenerry MR, Crosson B, DeBoe J and Leber WR (1988) STROOP Neuropsychological Screening Test Manual. Psychological Assessment Resources Inc., Florida.

Warrington EK (1984) Recognition Memory Test. Nelson, Windsor.

Wechsler D (1981) Wechsler Adult Intelligence Scale-Revised. The Psychological Corporation, New York.

Wilson BA, Cockburn JM, Baddeley AD and Hiorns R (1989) The development and validation of a test battery for detecting and monitoring everyday memory problems. Journal of Clinical and Experimental Neuropsychology, 11, 855-870.

Wright S, Young AW and Hellawell DJ (1993) Sequential Cotard and Capgras delusions. British Journal of Clinical Psychology, 321, 345-349.

Young AW, Reid I, Wright S and Hellawell DJ (1993) Face-processing impairments and the Capgras delusion. British Journal of Psychiatry, 162, 695-698.

(Received February 1994; accepted as revised 25 August 1994) 


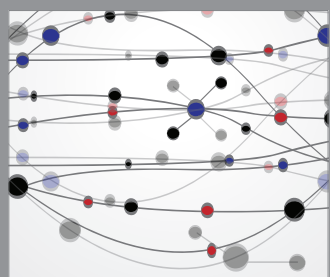

The Scientific World Journal
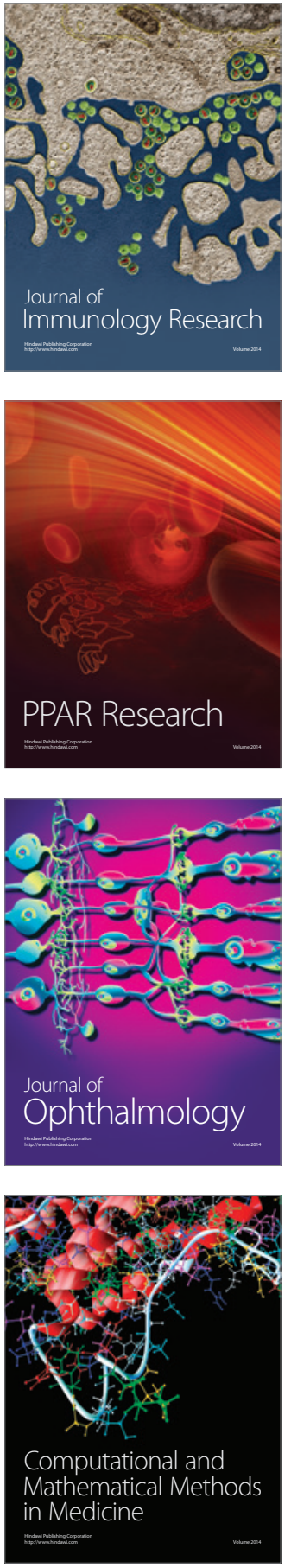

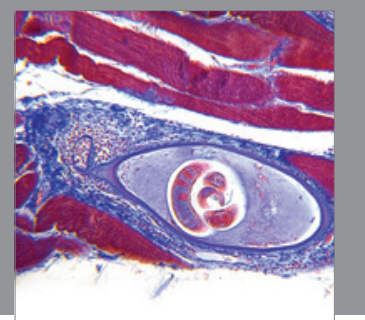

Gastroenterology

Research and Practice
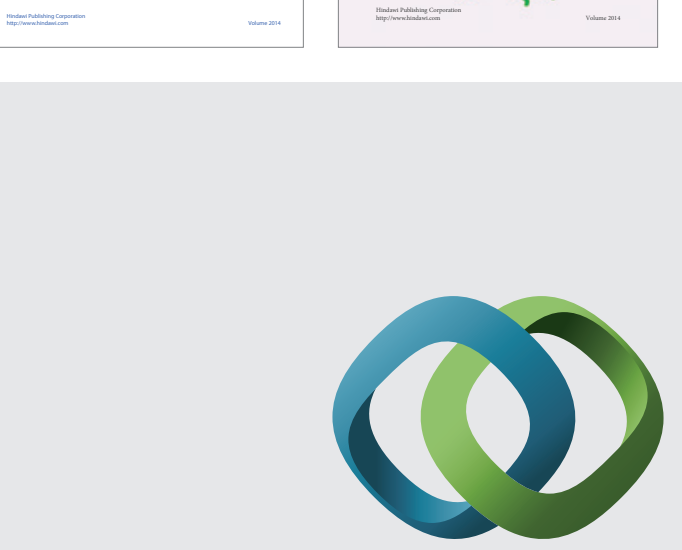

\section{Hindawi}

Submit your manuscripts at

http://www.hindawi.com
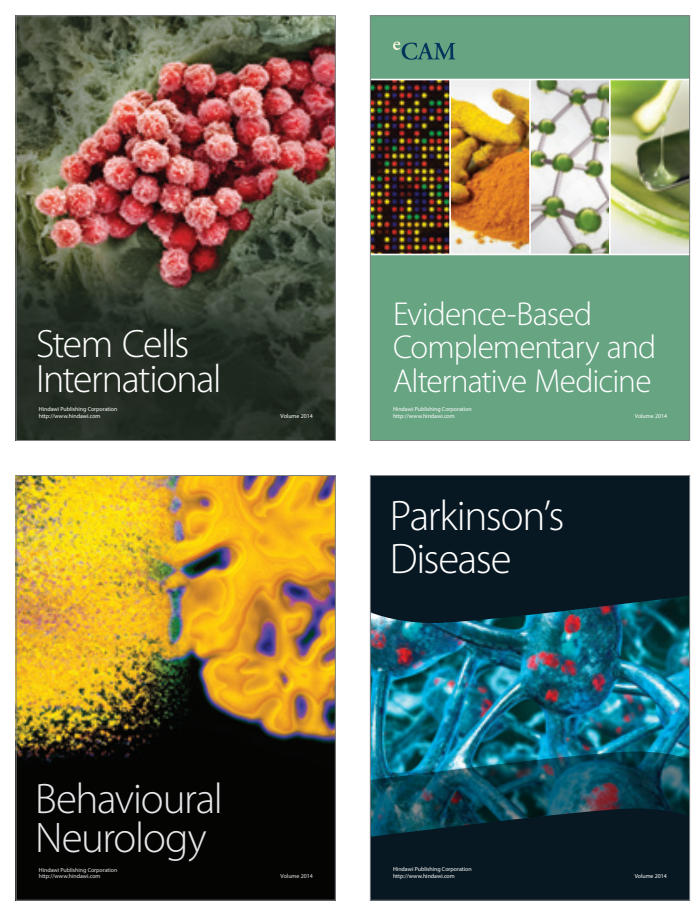

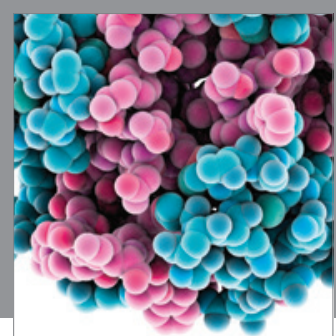

Journal of
Diabetes Research

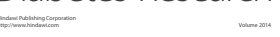

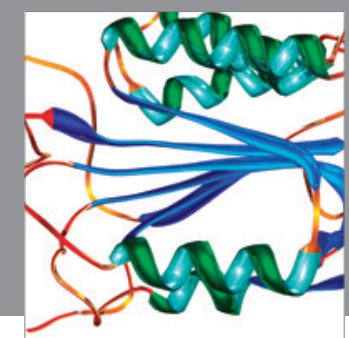

Disease Markers
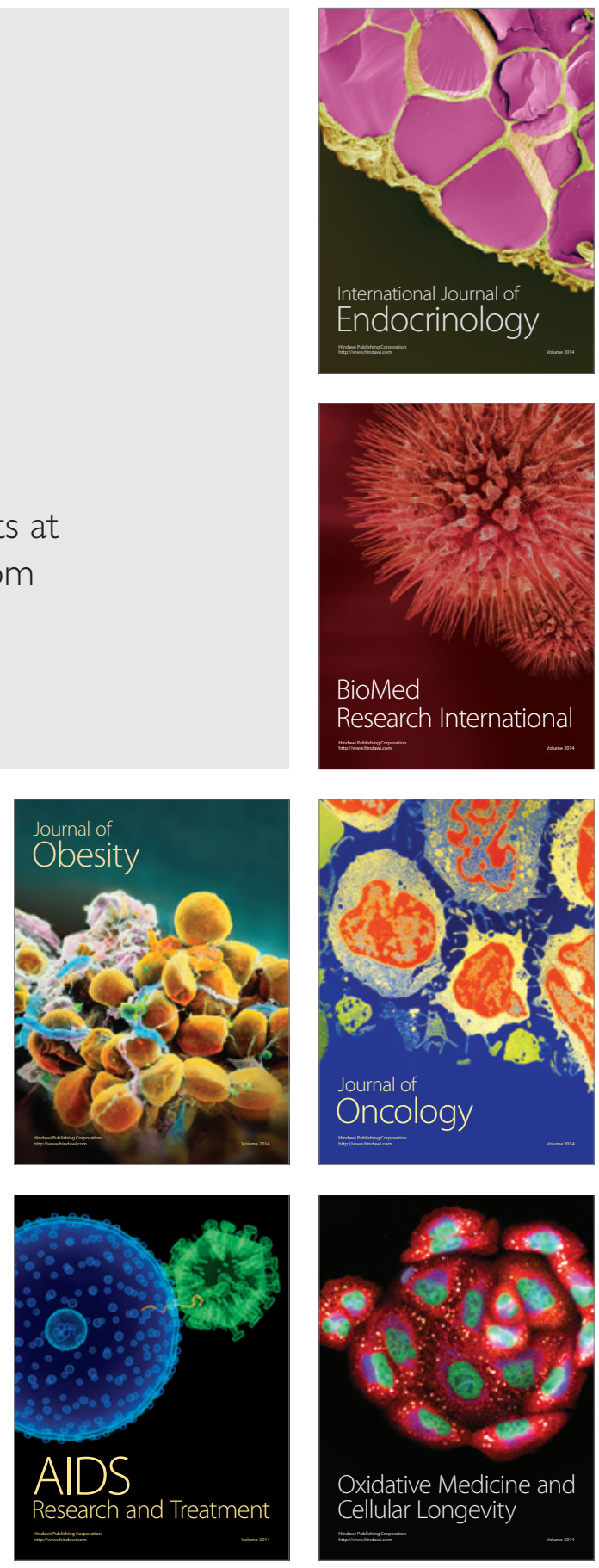